Research Article

\title{
Dmp53, basket and drICE gene knockdown and polyphenol gallic acid increase life span and locomotor activity in a Drosophila Parkinson's disease model
}

\author{
Hector Flavio Ortega-Arellano, Marlene Jimenez-Del-Rio and Carlos Velez-Pardo \\ Neuroscience Research Group, Medical Research Institute, Faculty of Medicine, University of Antioquia, \\ Medellin, Colombia.
}

\begin{abstract}
Understanding the mechanism(s) by which dopaminergic (DAergic) neurons are eroded in Parkinson's disease (PD) is critical for effective therapeutic strategies. By using the binary tyrosine hydroxylase (TH)-Gal4/UAS-X RNAi Drosophila melanogaster system, we report that Dmp53, basket and drICE gene knockdown in dopaminergic neurons prolong life span ( $p<0.05$; log-rank test) and locomotor activity $\left(p<0.05 ; \chi^{2}\right.$ test) in $D$. melanogaster lines chronically exposed to $(1 \mathrm{mM})$ paraquat ( $\mathrm{PQ}$, oxidative stress (OS) generator) compared to untreated transgenic fly lines. Likewise, knockdown flies displayed higher climbing performance than control flies. Amazingly, gallic acid (GA) significantly protected DAergic neurons, ameliorated life span, and climbing abilities in knockdown fly lines treated with $P Q$ compared to flies treated with $P Q$ only. Therefore, silencing specific gene(s) involved in neuronal death might constitute an excellent tool to study the response of DAergic neurons to OS stimuli. We propose that a therapy with antioxidants and selectively "switching off" death genes in DAergic neurons could provide a means for pre-clinical PD individuals to significantly ameliorate their disease condition.
\end{abstract}

Keywords: Basket, Dmp53, Drice, Drosophila, paraquat.

Received: June 14, 2013; Accepted: October 8, 2013.

\section{Introduction}

Parkinson's disease (PD) is a common progressive neurodegenerative disorder affecting millions of people worldwide (Alves et al., 2008). This neurological condition is clinically characterized by motor disorders (Jankovic, 2008). Pathologically it is prominently characterized by progressive loss of $50-70 \%$ of dopaminergic (DAergic) neurons located in the substantia nigra, abnormal protein aggregation, oxidative stress (OS) and mitochondrial dysfunction (Zhou et al., 2008; Cuervo et al., 2010; Xie et al., 2010). Despite intense investigation, the molecular mechanism(s) of cell loss is not yet fully established for target therapeutic strategies (Dexter and Jenner, 2013). This unmet need might partially explain the failure to establish definitive anti-Parkinson drugs (Rodnitzky, 2012). Since replacement of deficient dopamine constitutes a first-line symptomatic treatment (Gazewood et al., 2013), there is an urgent need to identify the molecular components involved in the DAergic neuronal demise.

Send correspondence to Carlos Velez-Pardo. Neuroscience Research Group, Medical Research Institute, Faculty of Medicine, University of Antioquia (UdeA), Calle 70 No. 52-21, and Calle 62 No. 52-59, Building 1, Room 412, SIU, Medellin, Colombia. E-mail: carlos.velez@neurociencias.udea.edu.co.
Paraquat (PQ, methyl viologen dichloride; or 1,10dimethyl-4,40-bipyridinium dichloride) is among the most consistently associated environmental risk factors for PD (Tanner et al., 2011; Wang et al., 2011). PQ might cause neuronal deterioration via OS and mitochondrial damage (Franco et al., 2010). Because ethical and policy issues limitation in human research, most of the PQ toxic effects have been studied in in vitro and in vivo models of PD. Specifically, our research group has shown that PQ induces apoptosis - a type of programed cell death - by an OSmediated mechanism (Jimenez-Del-Rio and Velez-Pardo, 2008). The major molecular events involve generation of $\mathrm{O}_{2}{ }^{--} / \mathrm{H}_{2} \mathrm{O}_{2}$, activation of the transcription factor $\mathrm{p} 53$, JNK (c-Jun N-terminal) kinase, mitochondria depolarization, caspase-3 activation and chromatin condensation/DNA fragmentation. Drosophila melanogaster has been used as biological tool to inquire on PD process (for a review see Muñoz-Soriano et al., 2011). Remarkably, PQ selectively destroys DAergic neurons in the fly (Chaudhuri et al., 2007), via OS and mitochondrial damage (Bonilla et al., 2006; Hosamani and Muralidhara, 2013). In agreement with this data, Vrailas-Mortimer et al. (2012) have shown that some commercially available antioxidant supplements confer significant protection to D. melanogaster against PQ and $\mathrm{H}_{2} \mathrm{O}_{2}$. Furthermore, we have recently shown that 
polyphenols [e.g., gallic acid (GA), propyl gallate (PG), epicatechin (EC)], which are well-known antioxidant and gene modulators, were able to protect, rescue and restore impaired movement activity in Drosophila induced by acute (Jimenez-Del-Rio et al., 2010) or chronic (OrtegaArellano et al., 2011) PQ exposure. It has also been shown that pharmacological inhibition of JNK increased life span and locomotor activity (i.e., climbing) compared to flies treated with PQ (Jimenez-Del-Rio et al., 2008). Taken together, these findings suggest that polyphenols might modulate life span and movement capabilities in $D$. melanogaster exposed to $\mathrm{PQ}$, and that JNK signalling might be involved in those effects. Since there is compelling evidence that the apoptosis pathway is conserved between Drosophila and mammalian cells (Oberst et al., 2008; O'Riordan et al., 2008; Mollereau, 2009), and essential signalling molecules involved in the OS response in mammalian cells, such as p53, JNK and caspase-3 are highly similar to Drosophila Dmp53 (43\%), basket (87\%) and $\operatorname{drICE}(61 \%)$, respectively (Reiter et al., 2001; Chien et al., 2002), our hypothesis is that a decreased gene expression of Dmp53, basket and drICE in DAergic neurons might have a beneficial impact on flies exposed to PQ in terms of DAergic neurons survival, life span and climbing capabilities. Moreover, transgenic flies treated with antioxidants might show even longer life span and functionality than transgenic flies treated with PQ alone.

To evaluate this premise, a genetically amenable binary tyrosine hydroxylase (TH)-Gal4/upstream activator sequences (UAS-X) RNA interference (RNAi) $D$. melanogaster system was used, wherein each gene/protein (X) can be silenced by RNAi (Kennerdell and Carthew, 2000) in DAergic neurons. RNAi provides an easy and powerful technique to suppress gene expression by specific removal of mRNA molecules transcribed from endogenous genes (Dietzl et al., 2007). Therefore, RNAi could result in a potent targeted therapeutic strategy in PD (Gavrilov and Saltzman, 2012). Although systematic RNAi analyses have revealed the role of cell death machinery components (e.g., caspases, and caspase-adaptors) in Drosophila eye development (Leulier et al., 2006), and JNK and caspase-3 RNAi experiments were done in wing imaginal discs (Umemori et al., 2009), no data is yet available to determine whether Dmp53, basket or drICE RNAi have any impact on life span or movement activity in the fly.

The aims of the present investigation were to study the life span and locomotor activity of Dmp53, basket and drICE gene knockdown in D. melanogaster (e.g., TH-Gal4 ${ }^{+/}$, UAS-Dmp53 $\mathrm{RNAi}^{+/}$; $\mathrm{TH}$-Gal4 ${ }^{+/}$, UAS-basket $\mathrm{RNAi}^{+/}$; $\mathrm{TH}-\mathrm{Gal4}^{+/}$, UAS-drICE $\mathrm{RNAi}^{+/}$) chronically exposed to $1 \mathrm{mM} P Q$ added to a $1 \%$ glucose feeding regimen for 15 days, and to determine whether polyphenols such as GA affect life span and locomotor activity of transgenic flies exposed to PQ for 15 days. GA was selected based on its effectiveness against PQ toxicity (Jimenez-Del-Rio et al., 2010; Ortega-Arellano et al., 2011). Taken together, our findings suggest that PQ induces DAergic neuronal deterioration in Drosophila by a p53-, JNK- and caspase3-dependent mechanism similar to that seen in human cells (Jimenez-Del-Rio and Velez-Pardo, 2012). It is inferred that silencing specific gene(s) involved in neuronal cell death might constitute an excellent tool to study the response of DAergic neurons to OS stimuli. Given that GA proved to be an effective antioxidant against PQ, polyphenols therefore become an important source for longterm prophylactic applications. Understanding the relevant events of cell death in DAergic neurons is a necessary step to better establish genetic and/or antioxidant therapy approaches in PD. Consequently, we proposed that a therapy with antioxidants and selectively "switching off" of death genes in DAergic neurons should provide a means for pre-clinical PD individuals to significantly ameliorate their disease condition.

\section{Materials and Methods}

\section{Fly stocks and culture}

Wild type Canton-S and fly lines were cultured under standard conditions, as described elsewhere (Ortega-Arellano et al., 2011). The genotypes were established by standard genetics. Fly Stocks obtained from the Bloomington Stock Center (BSC) were: TH-Gal4 (\#8848), and UASGFP (\#1521). UAS-dsRNAi (double-stranded RNA interference) lines obtained from the Vienna Drosophila RNAi stock center (VDRC) were: UAS-drICE RNAi (\#28006), UAS-basket RNAi (\#34138), and UAS-Dmp53 RNAi (\#10692). Male TH-GAL4 ${ }^{++}$flies were crossed with wild type Canton-S females to obtain heterozygous female flies (fF1, TH-GAL4 $4^{+-}$, Figure 1A). Male $T H-G A L 4^{+/+}$flies were crossed with $U A S-X R N A i$ females (Figure 1B) to obtain heterozygous female flies (fF1) $\left(T H-G A L 4^{+-}, U A S-\right.$ Dimp53 $\left.R N A i^{+-}\right)$. Since female flies were shown to be particularly sensible to PQ (Jimenez-Del-Rio et al., 2008), the $\mathrm{fF} 1$ generation was collected under brief $\mathrm{CO}_{2}$ anesthesia within 2 to 3 days after eclosion for further experiments.

\section{Paraquat toxicity assay}

The paraquat toxicity assay was performed on virgin 2- to 3-day-old fF1 flies collected overnight and kept on regular food medium. Subsequently, 50 separated adult $\mathrm{fF} 1$ flies were starved in empty vials for $3 \mathrm{~h}$ at $25{ }^{\circ} \mathrm{C}$. Then, groups of five flies were placed in ten vials containing a filter paper (Bio Rad Mini Trans-Blot 1703932) saturated with $1 \%$ glucose $(55.5 \mathrm{mM}$ glucose, Gluc) in distilled water $(\mathrm{dW})$ for $24 \mathrm{~h}$. After this time, flies were starved in empty vials for $3 \mathrm{~h}$ at $25^{\circ} \mathrm{C}$ and transferred to vials with a filter paper saturated with $200 \mu \mathrm{L}(1 \mathrm{mM}) \mathrm{PQ}$ in Gluc for 15 days. Filters were changed daily. Red food dye $(8 \mu \mathrm{L} / \mathrm{mL})$ (Red food colour McCormick) was added to ensure homogeneity and food intake. Living flies were counted daily. 

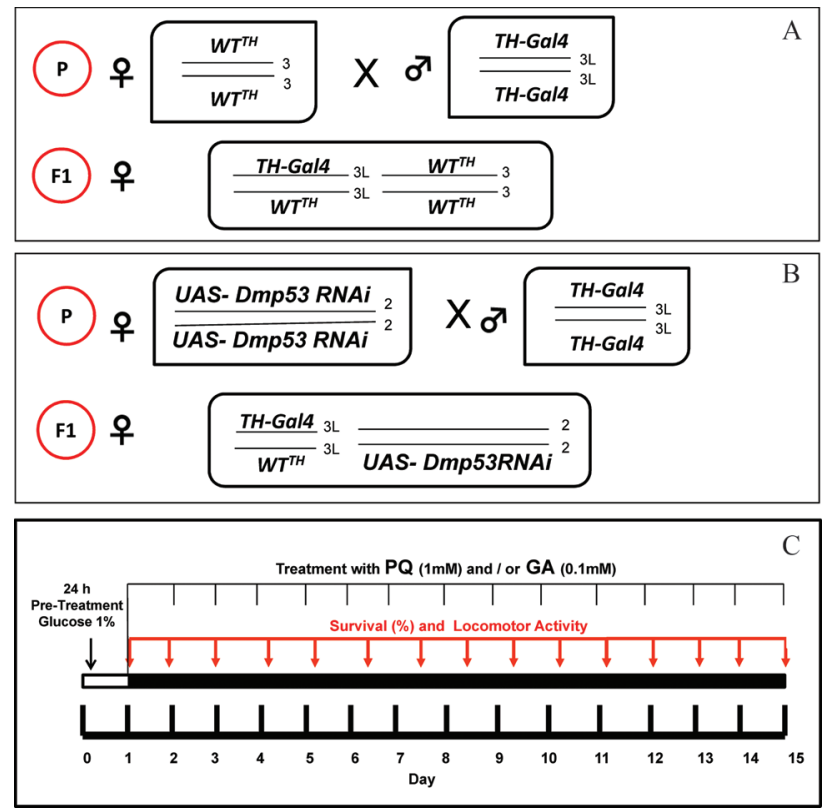

Figure 1 - Scheme for basic fly cross and selection. (A) $\mathrm{TH}-\mathrm{Gal}^{+/}$flies were obtained by crossing $\mathrm{TH}-\mathrm{Gal4}^{+/+}$(males, $\mathrm{n}=10$ ) and wild type Canton S Drosophila melanogaster (females, $\mathrm{n}=10$ ). After five days of husbandry, parental flies were discarded from the mating tubes. F1 flies were then reared according to standard procedures. (B) $T H-\mathrm{Gal}_{4}^{+/}, \mathrm{UAS}-\mathrm{X}$ $R N A i^{+/-}$flies, where $X$ represent either Dmp53, basket or drICE gene, were obtained by crossing $\mathrm{TH}_{\mathrm{Gal4}}^{+/+}$(males, $\mathrm{n}=10$ ) and $U A S-X \mathrm{RNAi}^{+{ }^{++}}$(females, $\mathrm{n}=10$ ). For the sake of clarity, $T H-\mathrm{Gal4}^{+/} U A S-D m p 53 \mathrm{RNAi}^{+/-}$ transgenic is shown as representative sample. (C) Schematic representation of feeding schedule, paraquat (PQ), and/or polyphenol (gallic acid, GA) treatments in Drosophila melanogaster.

\section{Antioxidant assay}

The antioxidant assay was also performed on virgin 2- to 3- day-old fF1 flies collected overnight and kept on regular food medium. Subsequently, 50 adult $\mathrm{fF} 1 \mathrm{flies}$ were starved as described above and groups of five flies were placed in ten vials containing a filter paper saturated with $1 \mathrm{G}$ in $\mathrm{dW}$ for $24 \mathrm{~h}$. Then, flies were fed for 15 days with $200 \mu \mathrm{L}$ Gluc solution containing $0.1 \mathrm{mM}$ fresh polyphenol solution (e.g., GA) and $1 \mathrm{mM}$ PQ. Filters were changed daily. Red food dye was added as described and survival proportion and locomotion assay (\%) were rated at each interval of time.

\section{Locomotion assay}

The movement deficit assay was performed on treated and untreated flies according to Ortega-Arellano et al. (2011). Briefly, untreated or treated $\mathrm{fF} 1$ flies were placed in empty plastic vials. After a 10 min resting period, the flies were tapped to the bottom of the vials and the number of flies able to climb $5 \mathrm{~cm}$ in $6 \mathrm{~s}$ was recorded at each time interval. The assays were repeated three times at $1 \mathrm{~min}$ intervals. For each experiment, a climbing percent (\%) was calculated, defined as $1 / 2\left[\left(\mathrm{n}_{\text {tot }}+\mathrm{n}_{\text {top }}-\mathrm{n}_{\text {bot }}\right) / \mathrm{n}_{\text {tot }}\right] \times 100$. Data were shown as mean \pm standard deviation of the mean (SD).
A Chi Square $\left(\chi^{2}\right)$ test was performed to compare the proportion of percentage between independent groups. Differences were considered statistically significant at $\mathrm{p}<0.05$.

\section{Survival test}

fF1 flies were treated chronically with PQ and polyphenol (GA) as described above for 15 days. Live flies were counted daily in groups of five flies per vial. 50 flies per treatment were used. Survival curves were plotted using the Kaplan-Meier estimator. The statistical significance was calculated using the log rank test implemented in the portable IBM SPSS statistics 19 package program. The null hypothesis in all survival assays was that the exposure of genetically modified Drosophila to PQ and/or GA made no difference to the survival of the flies in the absence of these reagents. Differences were considered statistically significant at $\mathrm{p}<0.05$.

\section{Results}

The knockdown of Dmp53, basket and drICE genes in dopaminergic neurons increases life span and locomotor activity in Drosophila melanogaster exposed to paraquat

We initially wanted to evaluate life span and locomotor activity in D. melanogaster TH-Gal4 ${ }^{+/}$, (Figure 1A, control) resulting from the cross between wild type Canton $S$ and homozygous $\mathrm{TH}-\mathrm{Gal4}^{+/+}$, exposed to $1 \mathrm{mM}$ PQ in the experimental design (Figure 1C). As shown in Figure 2A, the proportion of surviving $\mathrm{TH}-\mathrm{Gal4}^{+/}$flies treated with PQ was significantly diminished compared to $\mathrm{TH}-\mathrm{Gal4}^{+/}$ flies. Indeed, while $50 \%$ of the $\mathrm{TH}-\mathrm{Gal}^{+/-}$flies perished by day 14 , the $\mathrm{TH}-\mathrm{Gal4}^{+/}$plus PQ group did so at day 5. The percentage of locomotor activity (i.e., $>75 \%$ climbing performance) remained normal in $\mathrm{TH}-\mathrm{Gal4}^{+/-}$flies until day 15 , whereas climbing performance was drastically diminished already by day 5 when they were exposed to PQ (Figure 2B). Interestingly, knockdown flies $\mathrm{TH}-\mathrm{Gall}^{++}$, UAS-Dmp53 RNAi ${ }^{+/+}$(Figure $2 \mathrm{~A}, \mathrm{~B}$ ); $\mathrm{TH}_{-\mathrm{Gal4}}{ }^{+-}, \mathrm{UAS}$ bsk $\mathrm{RNAi}^{+/}$(Figure $3 \mathrm{~A}, \mathrm{~B}$ ), and $\mathrm{TH}-\mathrm{Gal4}^{+/}, \mathrm{UAS}$ - drICE $R N A i^{+-}$(Figure $4 \mathrm{~A}, \mathrm{~B}$ ) displayed survival percentages and climbing capabilities comparable to control flies ( $T H-$ $\mathrm{Gal4}^{+/}$). However, in $50 \%$ of the transgenic flies treated with PQ the percentage of survival slightly increased (by 2 days) and climbing performance moderately augmented (by 3-5 days) compared to the control group (Figures 2, 3 and $4 \mathrm{~A}, \mathrm{~B})$.

\section{Gallic acid (GA) increases life span and locomotor activity in RNAi fly lines}

Polyphenols have shown a high protective effect against PQ in Drosophila (Jimenez-Del-Rio et al., 2010). We, thus, investigated herein whether GA also had an effect in genetically modified flies when exposed to $1 \mathrm{mM}$ PQ for 

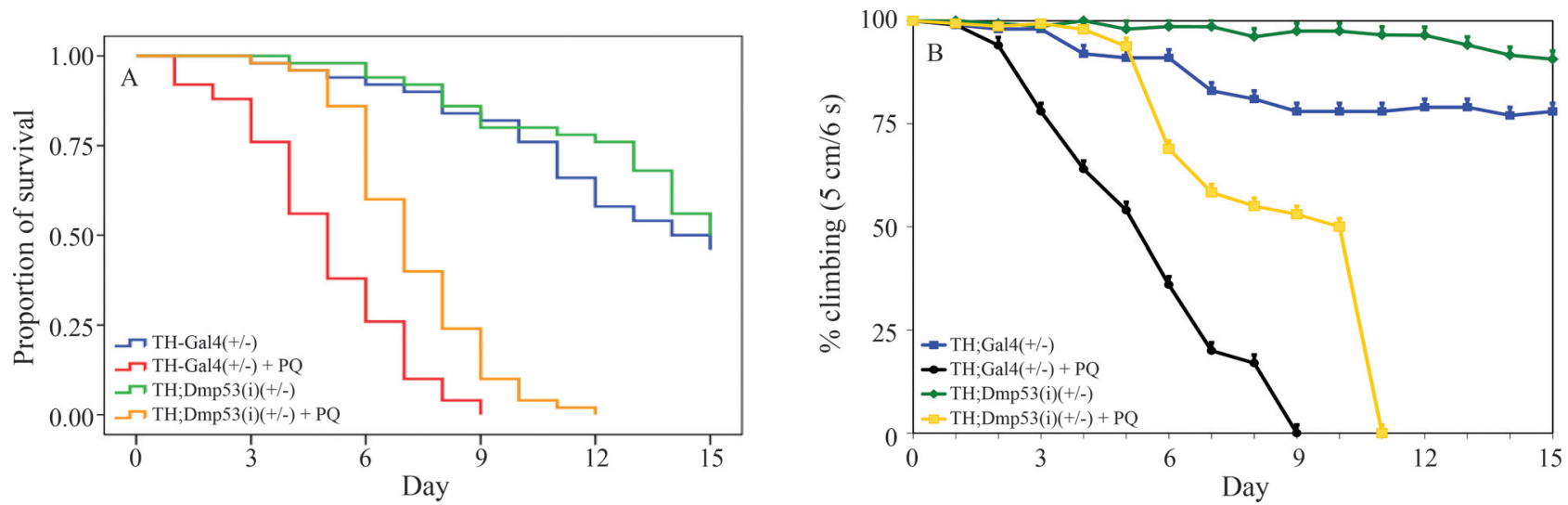

Figure 2 - Dmp 53 gene knockdown increases life span and locomotor activity in Drosophila melanogaster exposed to paraquat. Female flies ( $\mathrm{n}=50$ per treatment) were treated as described in Materials and Methods. The graphs show that the proportion of survival (A) and climbing performance (B) were significantly increased in $\mathrm{TH}-\mathrm{Gal4}^{+/}, \mathrm{UAS}-\mathrm{Dmp} 53 \mathrm{RNAi}^{+/}$gene knockdown flies compared to the $\mathrm{TH}-\mathrm{Gal4}^{+/-}$fly line (control). Statistical comparisons between untreated and treated flies showed: (A) a $\mathrm{p}<0.05$ by log-rank test and (B) a $\mathrm{p}<0.05$ by $\chi^{2}$ test. Comparisons between treated gene knockdown flies and the $\mathrm{TH}-\mathrm{Gall}^{+/}$fly line showed statistical significance.

15 days. As shown in Figure 5, the presence of GA in the diet of $\mathrm{TH}-\mathrm{Gal4}^{+/}$, UAS-Dmp $53 \mathrm{RNAi}^{+/}$fly lines treated with PQ increased the proportion of survival (Figure 5A) and climbing performance (Figure 5B) compared to flies treated with PQ alone. Noticeably, while $50 \%$ of the Dmp $53 R N A i^{+/}$flies treated with PQ and GA survived when scored on days 10 and 13 , respectively, $50 \%$ survival in the $\operatorname{Dmp} 53 R \mathrm{RAi}^{+/}$fly line treated with PQ alone could only be scored until day 7 and 10 , respectively. Similar results were obtained with the other RNAi transgenic flies (Table 1).

\section{Discussion}

By using a GAL4/UAS-X RNAi system, we could show that Dmp53, basket and $d r I C E$ gene knockdown prolonged life span ( $\mathrm{p}<0.05, \log$ rank test) and locomotor activity (i.e. climbing capability, $\mathrm{p}<0.05, \chi^{2}$ test) in $D$.

Table 1 - Dmp53, bsk and drICE gene knockdown increase the life span and locomotor activity of Drosophila melanogaster chronically exposed to paraquat.

\begin{tabular}{|c|c|c|c|c|c|c|c|}
\hline \multirow[t]{2}{*}{ Line } & \multirow{2}{*}{$\begin{array}{l}\text { Noxious/ } \\
\text { antioxidant }\end{array}$} & \multirow[t]{2}{*}{ Treatment } & \multirow{2}{*}{$\begin{array}{l}\text { Concentration } \\
(\mathrm{mM})\end{array}$} & \multicolumn{4}{|c|}{ Effect on } \\
\hline & & & & $\begin{array}{c}\text { Survival } \\
(50 \%)^{\mathrm{a}}\end{array}$ & $\mathrm{K}-\mathrm{M}, \mathrm{p}$ & $\begin{array}{c}\text { Climbing } \\
(50 \%)^{\mathrm{b}}\end{array}$ & $\chi^{2}, p$ \\
\hline \multirow[t]{4}{*}{$T H-G A L 4^{+/-}$} & PQ & 1 & $\mathbf{0}$ & $14 \pm 0.6$ & 1 vs. $2, \mathrm{p}<0.005$ & $>15$ & 1 vs. $2, \mathrm{p}<0.05$ \\
\hline & $\mathbf{P Q}$ & 2 & 1 & $5 \pm 0.3$ & 2 vs. $4, \mathrm{p}<0.005$ & 5 & 2 vs. $4, \mathrm{p}<0.05$ \\
\hline & GA & 3 & 0.1 & 15 & 3 vs. $4, \mathrm{p}<0.005$ & $>15$ & 3 vs. $4, \mathrm{p}<0.05$ \\
\hline & GA + PQ & 4 & $0.1+1$ & $11 \pm 0.4$ & 4 vs. 812 16, n.s. & 13 & 4 vs. 812 16, n.s. \\
\hline \multirow{4}{*}{$\begin{array}{l}\text { UAS D Dmp 53(RNAi) } \\
\text { TH-Gal4 }\end{array}$} & PQ & 5 & $\mathbf{0}$ & $15 \pm 0.4$ & 1 vs. 5 , n.s. & $>15$ & 1 vs. 5 , n.s \\
\hline & PQ & 6 & 1 & $7 \pm 0.4$ & 2 vs. $6, \mathrm{p}<0.005$ & 10 & $2 v s .6, \mathrm{p}<0.05$ \\
\hline & GA & 7 & 0.1 & 15 & 6 vs. $8, \mathrm{p}<0.005$ & $>15$ & 6 vs. $8, \mathrm{p}<0.05$ \\
\hline & $\mathbf{G A}+\mathbf{P Q}$ & 8 & $0.1+1$ & $10 \pm 0.5$ & 7 vs. $8, \mathrm{p}<0.005$ & 13 & 7 vs. $8, \mathrm{p}<0.05$ \\
\hline \multirow{4}{*}{$\begin{array}{l}\text { UAS } \sim \text { bsk }(R N A i)^{+/-} \\
\text {TH-Gal4 }\end{array}$} & PQ & 9 & $\mathbf{0}$ & $15 \pm 0.4$ & 1 vs. 9 , n.s. & $>15$ & 1 vs. 9 , n.s. \\
\hline & $\mathbf{P Q}$ & 10 & 1 & $7 \pm 0.5$ & 2 vs. $10, \mathrm{p}<0.005$ & 9 & 2 vs. $10, \mathrm{p}<0.05$ \\
\hline & GA & 11 & 0.1 & 15 & 10 vs. $12, \mathrm{p}<0.005$ & $>15$ & 10 vs. $12, \mathrm{p}<0.05$ \\
\hline & $\mathbf{G A}+\mathbf{P Q}$ & 12 & $0.1+1$ & $10 \pm 0.5$ & 11 vs. $12, \mathrm{p}<0.005$ & 13 & 11 vs. $12, \mathrm{p}<0.05$ \\
\hline \multirow{4}{*}{$\begin{array}{l}U A S \sim d r I C E(R N A i)^{+/-} \\
T H-G a l 4^{+/}\end{array}$} & PQ & 13 & $\mathbf{0}$ & 15 & 1 vs. 13 , n.s. & $>15$ & 1 vs. 13 , n.s. \\
\hline & $\mathbf{P Q}$ & 14 & 1 & $7 \pm 0.5$ & 2 vs. $14, \mathrm{p}<0.005$ & 8 & 2 vs. $14, \mathrm{p}<0.05$ \\
\hline & GA & 15 & 0.1 & 15 & 14 vs. $16, \mathrm{p}<0.005$ & $>15$ & 14 vs. $16, \mathrm{p}<0.05$ \\
\hline & $\mathbf{G A}+\mathbf{P Q}$ & 16 & $0.1+1$ & $11 \pm 0.4$ & 15 vs. $16, \mathrm{p}<0.005$ & 13 & 15 vs. $16, \mathrm{p}<0.05$ \\
\hline
\end{tabular}

${ }^{a}$ represents number of days at which $50 \%$ of total flies have been killed.

${ }^{b}$ represents number of days at which $50 \%$ of climbing ability is impaired.

Abbreviations: Paraquat, PQ; Galic Acid, GA; K-M, Kaplan-Meier test; n.s., no significance; $\chi^{2}$, Chi-square test. 

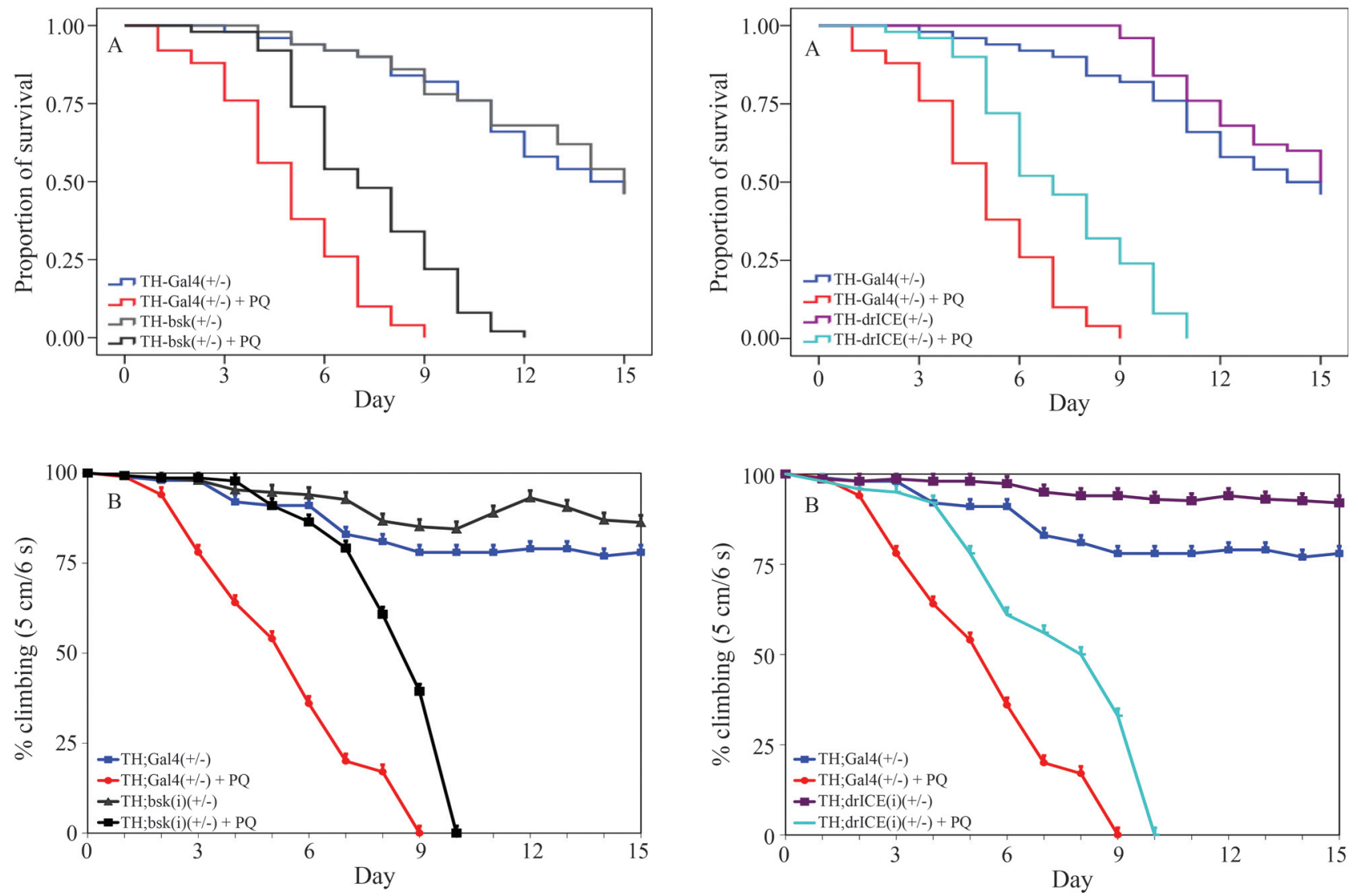

Figure 3 - Basket gene knockdown increases life span and locomotor activity in Drosophila melanogaster exposed to paraquat. Female flies $(\mathrm{n}=$ 50 per treatment) were treated as described in Materials and Methods. The graphs show that the proportion of survival and climbing performance were significantly increased in $\mathrm{TH}-\mathrm{Gal4}^{+/}, \mathrm{UAS}$-bsk $\mathrm{RNAi}^{+/}$gene knockdown flies compared to the $\mathrm{TH}-\mathrm{Gal}^{+/}$fly line (control). Statistical comparisons between untreated and treated flies showed: (A) a $p<0.05$ by log-rank test and (B) a $\mathrm{p}<0.05$ by $\chi^{2}$ test. Comparisons between treated gene knockdown flies and the $\mathrm{TH}-\mathrm{Gal4}^{+/-}$fly line showed statistical significance.

melanogaster lines chronically exposed to PQ compared to controls. These findings comply with the notion that altered gene function, either by mutation or knockdown can modulate the susceptibility to a known environmental PD risk factor such as PQ (Goldman et al., 2012). However, the PQ toxic effect was dependent on the genetic background of the exposed flies. One possible explanation for this finding is that while some DAergic neurons can cope with a rise in OS, others are more vulnerable (Wang and Michaelis, 2010). Because of such selective vulnerability, these neurons are usually the first to exhibit cell death and functional decline (i.e. climbing performance).

A second possibility is that some mutated (Goldman et al., 2012) or experimentally knocked down gene(s) may confer resistance to DAergic neurons against PQ-driven OS, thereby increasing life span and locomotor activity. Our data support the latter hypothesis. In fact, Dmp 53, basket and drICE RNAi described herein in an in vivo system appears to mirror the pharmacological inhibition of $\mathrm{p} 53$,

Figure 4 - DrICE gene knockdown increases life span and locomotor activity in Drosophila melanogaster exposed to paraquat. Female flies ( $\mathrm{n}=$ 50 per treatment) were treated as described in Materials and Methods. The graphs show that the proportion of survival and climbing performance were significantly increased in $\mathrm{TH}-\mathrm{Gal4}^{+/}$, UAS-drICE $\mathrm{RNAi}^{+/}$gene knockdown flies compared to the $\mathrm{TH}-\mathrm{Gal4}^{+/-}$fly line (control). Statistical comparisons between untreated and treated flies showed: (A) a $p<0.05$ by log-rank test and (B) a $\mathrm{p}<0.05$ by $\chi^{2}$ test. Comparisons between treated gene knockdown flies and the $\mathrm{TH}$-Gal4 ${ }^{+/}$fly line showed statistical significance.

JNK, caspase-3 and cell survival previously seen in vitro (Jimenez-Del-Rio and Velez-Pardo, 2008). Therefore, these data suggest that PQ induces a molecular mechanism of cell death in DAergic neurons that is similar in Drosophila melanogaster, mice (Peng et al., 2004) and primary human cells (Jimenez-Del-Rio and Velez-Pardo, 2008, 2012).

This conclusion is strongly supported by several observations. First, the Drosophila genome contains all the genes encoding canonical mitochondrial (Miwa et al., 2003; Oberst et al., 2008) and cell death proteins (Shi, 2004; O'Riordan et al., 2008; Steller, 2008), suggesting that they function in a similar manner as in mammalian cells (see O'Riordan et al., 2008). Indeed, the Drosophila Apaf-1 related killer (Dark) assembles in an apoptosome that functions within the intrinsic cell death pathway similar to that seen in mammals (Yuan et al., 2011). During apoptosis, the initiator caspase Dronc (ortholog of the mammalian pro-caspase-9) is activated by the Dark 

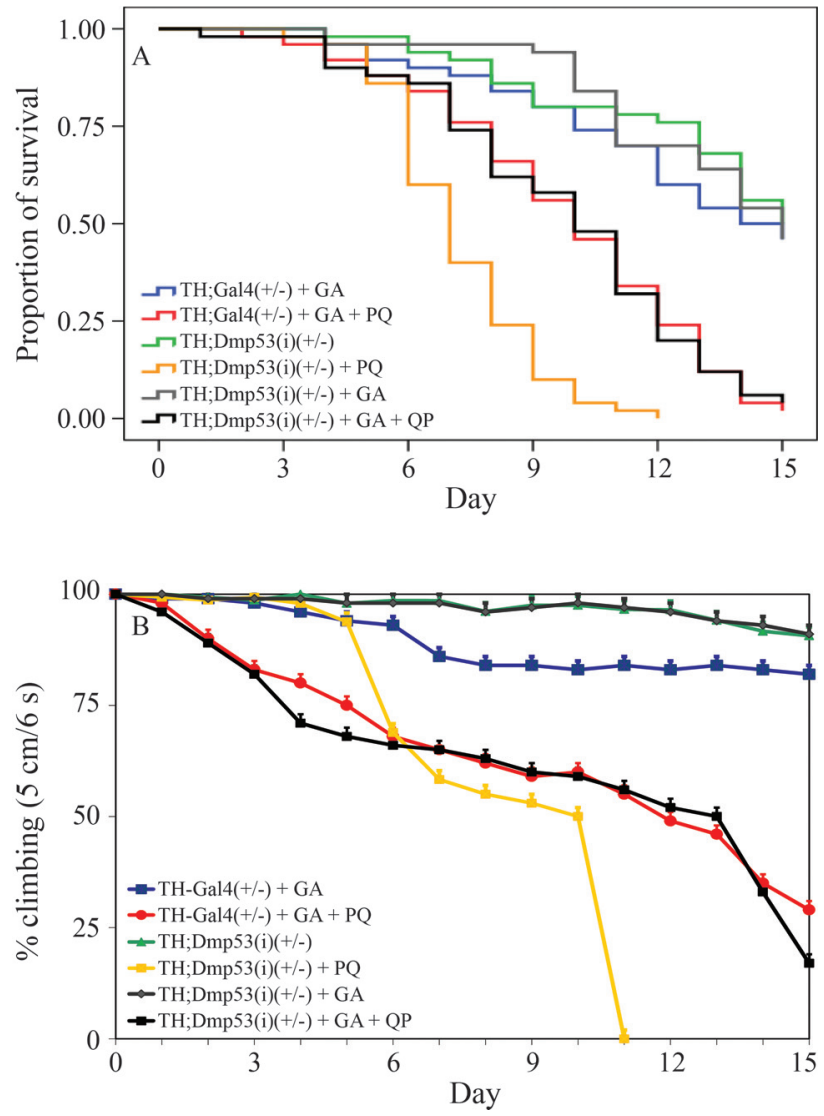

Figure 5 - Gallic acid (GA) increases survival (A) and locomotor activity (B) in gene knockdown Drosophila melanogaster lines exposed to paraquat. Female flies ( $\mathrm{n}=50$ per treatment) were treated as described in Materials and Methods. The graphs show that the proportion of survival (A) and climbing performance (B) were significantly increased in TH-Gal4 ${ }^{+/}, U A S-D m p 53 R N A i^{+/}$flies treated with $(1 \mathrm{mM})$ PQ and $(0.1 \mathrm{mM}) \mathrm{GA}$. Similar results were obtained with $\mathrm{TH}-\mathrm{Gal4}^{+/}, \mathrm{UAS}-\mathrm{bsk}$ $\mathrm{RNAi}^{+/}$and $\mathrm{TH}$-Gal4${ }^{+/}, \mathrm{UAS}$-drICE RNAi ${ }^{+/}$flies (Table 1). Statistical comparisons between untreated and treated flies showed: (A) a $p<0.05$ by log-rank test and (B) a p $<0.05$ by $\chi^{2}$ test. Comparisons between gene knockdown flies treated with GA plus PQ, and those treated with PQ alone showed statistical significance.

apoptosome. This allows Dronc to cleave the execution caspase DrICE (ortholog of the mammalian caspase-3), which, via proteolytic cleavage of the Drosophila inhibitor of caspase-activated DNase (dICAD, Mukae et al., 2000) or caspase-activated DNase (CAD, Yokoyama et al., 2000)), culminates in cell death. Second, stress-activated intracellular response signaling molecules (e.g. MAP kinase kinase kinase family proteins) are evolutionary conserved and appears to mirror their mammalian counterparts. Indeed, TAK1 (transforming growth factor $\beta$-activated kinase-1) activates JNK (Silverman et al., 2003) probably via D-MEKK1, the Drosophila ortholog of mammalian MEKK4/MTK1 (Ryabinina et al., 2006). Third, pharmacological treatment with the JNK inhibitor SP600125 has been demonstrated to protect and rescue flies against acute PQ intoxication (Jimenez-Del-Rio et al., 2008).
Interestingly, it has been shown that a Dmp53/JNK (basket)-dependent feedback amplification loop is essential for the apoptotic response to stress in Drosophila (Shlevkov and Morata, 2012). It is therefore reasonable to think that PQ might be able to trigger activation of Dmp53, basket and drICE via $\mathrm{O}_{2}^{-} / \mathrm{H}_{2} \mathrm{O}_{2}$ signaling and cell death (Steller, 2008) in a similar fashion as proposed in mammalian cells (Jimenez-Del-Rio and Velez-Pardo, 2012). In accordance with this view, several reports have shown that PQ induces activation of JNK associated with OS and cell death in vitro and in vivo in mammalian DAergic neurons (Chun et al., 2001; Peng et al., 2004; Klintworth et al., 2007; Ramachandiran et al., 2007; Choi et al., 2010; NisoSantano et al., 2010). But these observations stand in contradiction with others that have shown that overexpression of JNK (Wang et al., 2003; Inamdar et al., 2012) or overexpression of JNK target genes, such as human peroxiredoxin II (hPrxII) and Jafrac1 (a Drosophila homolog of hPrxII) (Lee et al., 2009) confer protection against PQ-induced toxicity in DAergic neurons of the fly. The reason for these contradictory observations is not yet known. Therefore, further research will be required to clarify this issue.

Finally, PQ induces PD-like clinical features in Drosophila, including resting tremor, bradykinesis, and postural instability with or without neuronal damage. A similar PD-like phenotype can be generated by pharmacological inhibition of the tyrosine hydroxylase enzyme with $\alpha$-MT (alpha-methyl-tyrosine). In these experiments no neuronal damage was observed, but functional impairment (climbing performance) was evident (Bonilla-Ramirez et al., 2011).

In conclusion, these data suggest that the knockdown of specific gene(s) in the Drosophila brain may provide basic information about the mechanism(s) of cell death/survival and clinical behavior in human PD.

Polyphenols have been postulated as potential neuroprotectant molecules in neurodegenerative disorders including PD (Albarracin et al., 2012). In agreement with others (Long et al., 2009; Peng et al., 2011) and our previous investigations (Jimenez-Del-Rio et al., 2010; OrtegaArellano et $a$ l., 2011), we found that GA prolongs life span and climbing activity in wild type or knocked down $D$. melanogaster. These data suggest that GA (and probably other polyphenols) may protect DAergic neurons independently of the genetic background of the fly, either via direct interaction with ROS, enzymes, receptors and/or transcription factors (Fraga et al., 2010), or through antioxidant gene regulatory mechanisms (Kim et al., 1997; Peng et al., 2011). Our findings suggest that GA can protect DAergic neurons against PQ. Consequently, this polyphenol is capable of modifying the life span and locomotor capabilities of flies exposed to OS stimuli.

In summary, induced RNAi of specific pro-apoptotic genes in DAergic neurons in D. melanogaster increased survival and climbing performance under PQ treatment. 
Since polyphenols such as GA displayed antioxidant capacity, they may certainly contribute to develop nutritional strategies oriented towards preventing PD. Our data suggest that pharmacologically targeted proteins or knockdown of critical death signaling genes, such p53, JNK and caspase-3 in DAergic neurons, together with antioxidant exposure, may retard neural deterioration or neuronal loss, thereby, restoring or prolonging locomotor activity in PD patients. In addition, our in vivo data on flies link directly to in vitro work done on OS-induced cell death in mammalian cells (Shi, 2004; O'Riordan et al., 2008; Steller, 2008; Jimenez-Del-Rio and Velez-Pardo, 2012). Understanding the mechanism(s) by which dopaminergic neurons are eroded in PD is critical for the development of effective therapeutic strategies.

\section{Acknowledgments}

This work was supported by CODI grants \#2545 to CV-P \& MJ-Del-Rio. HFOA is a doctoral student in the Graduate Biology Program at UdeA and was funded by a Young Research Award from Colciencias contract \#8790018-2011 and Programa de Sostenibilidad 2011/2012 del Grupo Malaria-UdeA.

\section{References}

Albarracin SL, Stab B, Casas Z, Sutachan JJ, Samudio I, Gonzalez J, Gonzalo L, Capani F, Morales L and Barreto GE (2012) Effects of natural antioxidants in neurodegenerative disease. Nutr Neurosci 15:1-9.

Alves G, Forsaa EB, Pedersen KF, Dreetz-Gjerstad M and Larsen JP (2008) Epidemiology of Parkinson's disease. J Neurol 255:18-32.

Bonilla E, Medina-Leendertz S, Villalobos V, Molero L and Bohórquez A (2006) Paraquat induced oxidative stress in Drosophila melanogaster: Effects of melatonin, glutathione, serotonin, minocycline, lipoic acid and ascorbic acid. Neurochem Res 31:1425-1432.

Bonilla-Ramirez L, Jimenez-Del-Rio $\mathrm{M}$ and Velez-Pardo $\mathrm{C}$ (2011) Acute and chronic metal exposure impairs locomotion activity in Drosophila melanogaster: A model to study Parkinsonism. Biometals 24:1045-1057.

Chaudhuri A, Bowling K, Funderburk C, Lawal H, Inamdar A, Wang Z and O'Donnell JM (2007) Interaction of genetic and environmental factors in a Drosophila Parkinsonism model. J Neurosci 27:2457-2467.

Chien S, Reiter LT, Bier E and Gribskov M (2002) Homophila: Human disease gene cognates in Drosophila. Nucleic Acids Res 30:149-151.

Choi WS, Abel G, Klintworth H, Flavell RA and Xia Z (2010) JNK3 mediates paraquat- and rotenone-induced dopaminergic neuron death. J Neuropathol Exp Neurol 69:511-520.

Chun HS, Gibson GE, DeGiorgio LA, Zhang H, Kidd VJ and Son JH (2001) Dopaminergic cell death induced by MPP(+), oxidant and specific neurotoxicants shares the common molecular mechanism. J Neurochem 76:1010-1021.

Cuervo AM, Wong ES and Martinez-Vicente M (2010) Protein degradation, aggregation, and misfolding. Mov Disord 25:S49-S54.
Dexter DT and Jenner P (2013) Parkinson disease: From pathology to molecular disease mechanisms. Free Radic Biol Med 62:132-144.

Dietzl G, Chen D, Schnorrer F, Su KC, Barinova Y, Fellner M, Gasser B, Kinsey K, Oppel S, Scheiblauer S, et al. (2007) A genome-wide transgenic RNAi library for conditional gene inactivation in Drosophila. Nature 448:151-156.

Fraga CG, Galleano M, Verstraeten SV and Oteiza PI (2010) Basic biochemical mechanisms behind the health benefits of polyphenols. Mol Aspects Med 31:435-445.

Franco R, Li S, Rodriguez-Rocha H, Burns M and Panayiotidis MI (2010) Molecular mechanisms of pesticide-induced neurotoxicity: Relevance to Parkinson's disease. Chem Biol Interact 188:289-300.

Gavrilov K and Saltzman WM (2012) Therapeutic siRNA: Principles, challenges, and strategies. Yale J Biol Med 85:187200.

Gazewood JD, Richards DR and Clebak K (2013) Parkinson disease: An update. Am Fam Physician 87:267-273.

Goldman SM, Kamel F, Ross GW, Bhudhikanok GS, Hoppin JA, Korell M, Marras C, Meng C, Umbach DM, Kasten M, et al. (2012) Genetic modification of the association of paraquat and Parkinson's disease. Mov Disord 27:1652-1658.

Hosamani R and Muralidhara (2013) Acute exposure of Drosophila melanogaster to paraquat causes oxidative stress and mitochondrial dysfunction. Arch Insect Biochem Physiol 83:25-40.

Inamdar AA, Chaudhuri A and O'Donnell J (2012) The protective effect of minocycline in a paraquat-induced Parkinson's disease model in Drosophila is modified in altered genetic backgrounds. Parkinson's Dis 938528.

Jankovic J (2008) Parkinson's disease: Clinical features and diagnosis. J Neurol Neurosurg Psychiatry 79:368-376.

Jimenez-Del-Rio M and Velez-Pardo C (2008) Paraquat induces apoptosis in human lymphocytes: Protective and rescue effects of glucose, cannabinoids and Insulin-like growth factor-1. Growth Factors 26:49-60.

Jimenez-Del-Rio M and Velez-Pardo C (2012) The bad, the good, and the ugly about oxidative stress. Oxid Med Cell Longev 163913.

Jimenez-Del-Rio M, Daza-Restrepo A and Velez-Pardo C (2008) The cannabinoid CP55,940 prolongs survival and improves locomotor activity in Drosophila melanogaster against paraquat: Implications in Parkinson's disease. Neurosci Res 61:404-411.

Jimenez-Del-Rio M, Guzman-Martinez C and Velez-Pardo C (2010) The effects of polyphenols on survival and locomotor activity in Drosophila melanogaster exposed to iron and paraquat. Neurochem Res 35:227-238.

Kennerdell JR and Carthew RW (2000) Heritable gene silencing in Drosophila using double-stranded RNA. Nat Biotechnol 18:896-898.

Kim SJ, Han D, Ahn BH and Rhee JS (1997) Effect of glutathione, catechin, and epicatechin on the survival of Drosophila melanogaster under paraquat treatment. Biosci Biotechnol Biochem 61:225-229.

Klintworth H, Newhouse K, Li T, Choi WS, Faigle R and Xia Z (2007) Activation of c-Jun N-terminal protein kinase is a common mechanism underlying paraquat- and rotenoneinduced dopaminergic cell apoptosis. Toxicol Sci 97:149162.

Lee KS, Iijima-Ando K, Iijima K, Lee WJ, Lee JH, Yu K and Lee DS (2009) JNK/FOXO-mediated neuronal expression of fly 
homologue of peroxiredoxin II reduces oxidative stress and extends life span. J Biol Chem 284:29454-29461.

Leulier F, Ribeiro PS, Palmer E, Tenev T, Takahashi K, Robertson D, Zachariou A, Pichaud F, Ueda R and Meier P (2006) Systematic in vivo RNAi analysis of putative components of the Drosophila cell death machinery. Cell Death Differ 13:1663-1674.

Long J, Gao H, Sun L, Liu J and Zhao-Wilson X (2009) Grape extract protects mitochondria from oxidative damage and improves locomotor dysfunction and extends lifespan in a Drosophila Parkinson's disease model. Rejuvenation Res 12:321-331.

Mao Z, Davis RL (2009) Eight different types of dopaminergic neurons innervate the Drosophila mushroom body neuropil: Anatomical and physiological heterogeneity. Front Neural Circuits 3:5.

Miwa S, St-Pierre J, Partridge L and Brand MD (2003) Superoxide and hydrogen peroxide production by Drosophila mitochondria. Free Radic Biol Med 35:938-948.

Mollereau B (2009) Cell death: What can we learn from flies? Editorial for the special review issue on Drosophila apoptosis. Apoptosis 14:929-934.

Mukae N, Yokoyama H, Yokokura T, Sakoyama Y, Sakahira H and Nagata S (2000) Identification and developmental expression of inhibitor of caspase-activated DNase (ICAD) in Drosophila melanogaster. J Biol Chem 275:21402-21408.

Muñoz-Soriano V and Paricio N (2011) Drosophila models of Parkinson's disease: Discovering relevant pathways and novel therapeutic strategies. Parkinson's Dis 520640.

Niso-Santano M, González-Polo RA, Bravo-San Pedro JM, Gómez-Sánchez R, Lastres-Becker I, Ortiz-Ortiz MA, Soler G, Morán JM, Cuadrado A and Fuentes JM (2010) Activation of apoptosis signal-regulating kinase 1 is a key factor in paraquat-induced cell death: Modulation by the Nrf2/Trx axis. Free Radic Biol Med 48:1370-1381.

Oberst A, Bender C and Green DR (2008) Living with death: The evolution of the mitochondrial pathway of apoptosis in animals. Cell Death Differ 15:1139-1146.

O'Riordan MX, Bauler LD, Scott FL and Duckett CS (2008) Inhibitor of apoptosis proteins in eukaryotic evolution and development: A model of thematic conservation. Dev Cell 15:497-508.

Ortega-Arellano H, Jimenez-Del-Rio $\mathrm{M}$ and Velez-Pardo $\mathrm{C}$ (2011) Life span and locomotor activity modification by glucose and polyphenols in Drosophila melanogaster chronically exposed to oxidative stress-stimuli: Implications in Parkinson's disease. Neurochem Res 36:1073-1086.

Peng C, Chan HY, Huang Y, Yu H and Chen ZY (2011) Apple polyphenols extend the mean lifespan of Drosophila melanogaster. J Agric Food Chem 59:2097-2106.

Peng J, Mao XO, Stevenson FF, Hsu M and Andersen JK (2004) The herbicide paraquat induces dopaminergic nigral apoptosis through sustained activation of the JNK pathway. J Biol Chem 279:32626-32632.

Ramachandiran S, Hansen JM, Jones DP, Richardson JR and Miller GW (2007) Divergent mechanisms of paraquat, $\mathrm{MPP}+$, and rotenone toxicity: Oxidation of thioredoxin and caspase-3 activation. Toxicol Sci 95:163-171.

Reiter LT, Potocki L, Chien S, Gribskov M and Bier E (2001) A systematic analysis of human disease-associated gene sequences in Drosophila melanogaster. Genome Res 11:1114-1125.
Rodnitzky RL (2012) Upcoming treatments in Parkinson's disease, including gene therapy. Parkinsonism Relat Disord 18 Suppl 1:S37-S40.

Ryabinina OP, Subbian E and Lordanov MS (2006) D-MEKK1, the Drosophila orthologue of mammalian MEKK4/MTK1, and hemipterous/D-MKK7 mediate the activation of D-JNK by cadmium and arsenite in Schneider cells. BMC Cell Biol 7:e7.

Shi Y (2004) Caspase activation, inhibition, and reactivation: A mechanistic view. Protein Sci 13:1979-1987.

Shlevkov E and Morata G (2012) A dp53/JNK-dependant feedback amplification loop is essential for the apoptotic response to stress in Drosophila. Cell Death Differ 19:451460.

Silverman N, Zhou R, Erlich RL, Hunter M, Bernstein E, Schneider D and Maniatis T (2003) Immune activation of NFkappaB and JNK requires Drosophila TAK1. J Biol Chem 278:48928-48934.

Steller H (2008) Regulation of apoptosis in Drosophila. Cell Death Differ 15:1132-1138.

Tanner CM, Kamel F, Ross GW, Hoppin JA, Goldman SM, Korell M, Marras C, Bhudhikanok GS, Kasten M, Chade AR, et al. (2011) Rotenone, paraquat, and Parkinson's disease. Environ Health Perspect 119:866-872.

Umemori M, Habara O, Iwata T, Maeda K, Nishinoue K, Okabe A, Takemura M, Takahashi K, Saigo K, Ueda R, et al. (2009) RNAi-mediated knockdown showing impaired cell survival in Drosophila wing imaginal disc. Gene Regul Syst Bio 3:11-20.

Vrailas-Mortimer A, Gomez R, Dowse H and Sanyal S (2012) A survey of the protective effects of some commercially available antioxidant supplements in genetically and chemically induced models of oxidative stress in Drosophila melanogaster. Exp Gerontol 47:712-722.

Wang A, Costello S, Cockburn M, Zhang X, Bronstein J and Ritz B (2011) Parkinson's disease risk from ambient exposure to pesticides. Eur J Epidemiol 26:547-555.

Wang MC, Bohmann D and Jasper H (2003) JNK signaling confers tolerance to oxidative stress and extends lifespan in Drosophila. Dev Cell 5:811-816.

Wang X and Michaelis EK (2010) Selective neuronal vulnerability to oxidative stress in the brain. Front Aging Neurosci 2:e12.

Xie W, Wan OW and Chung KK (2010) New insights into the role of mitochondrial dysfunction and protein aggregation in Parkinson's disease. Biochim Biophys Acta 1802:935-941.

Yokoyama H, Mukae N, Sakahira H, Okawa K, Iwamatsu A and Nagata S (2000) A novel activation mechanism of caspaseactivated DNase from Drosophila melanogaster. J Biol Chem 275:12978-12986.

Yuan S, Yu X, Topf M, Dorstyn L, Kumar S, Ludtke SJ and Akey CW (2011) Structure of the Drosophila apoptosome at $6.9 \AA$ resolution. Structure 19:128-140.

Zhou C, Huang Y and Przedborski S (2008) Oxidative stress in Parkinson's disease: A mechanism of pathogenic and therapeutic significance. Ann NY Acad Sci 1147:93-104.

Associate Editor: Klaus Hartfelder

License information: This is an open-access article distributed under the terms of the Creative Commons Attribution License, which permits unrestricted use, distribution, and reproduction in any medium, provided the original work is properly cited. 